\title{
The power of early interventions in families with preterm born children
}

The Tromsø Intervention Study on Preterms (TISP) started in 1999 and we may now present results on children's socio-emotional and behavioural development, parental wellbeing and perceptions of the children's quality of life until children's age of nine.

Infants born preterm from the northernmost part of Norway (birth weight $<2000$ grams) were randomly selected to a premature intervention group ( $\mathrm{PI}, \mathrm{N}=72)$ and a premature control group (PC, $N=74$ ); 75 healthy newborns formed a term control group (TR). The intervention, a modified version of The Mother-Infant Transaction Program (MITP-M), included eight hours of supervision for the mother (and father if present) the last week in the $\mathrm{NICU}$ and four home visits about seven, 14, 30 and 90 days after discharge. The aim of the MITP-M was to strengthen parents' enthusiasm for their child and help them recognise signs of children's organization versus need for regulatory support. Furthermore, to show parents appropriate interactions with the child that would minimize child disturbance and increase interactional satisfaction for all partners. All families were followed up through developmental tests of children and responses to questionnaires at children's age of six months, one, two, three, five, seven and nine years. The main aim was to analyze whether differences between the $\mathrm{Pl}$ and the PC group emerged as a possible consequence of the intervention. The PI and the PC group were in addition compared with the results of the TR group.

Longitudinal analyzes showed that the average prevalence of behavior problems varied according to the same pattern in the three study groups from two to nine years. PC mothers and PC fathers reported slightly more behavior problems than PI-parents who had received the intervention at all follow-ups, even not creating significant differences between $\mathrm{Pl}$ and PC. No longitudinal differences were detected in longitudinal behavior development between the intervention group and the term control group, but one was detected between the PC and the TR group. Fathers in the PC group reported a steeper increase in symptoms of anxiety in their preterm-born children from their age of five until nine years compared with fathers of term-born children.

Significant differences between the PI and the PC group became visible at children's age of seven and nine years where both parents and teachers reported significantly less attentional problems and better social competence, adaptability and school-related performances in the PI group. PI children were in average reported as similar to the TR group at the age of nine, both what came to school adjustment, achievements and the occurrence of problems. Significant differences between the PC and the TR group persisted concerning both behavior problems and competencies. The presentation will also document how the intervention may have strengthened parents and children's wellbeing. The research presented in this presentation indicate that the structured guidance given parents of preterms in the newborn period has promoted positive and long-lasting effects on the PI group as these children perform at similar level as their term-born peers at nine years of age. 